David Kleingers*

\title{
Bilder neu bewegen - Streaming als Baustein einer ganzheitlichen Strategie zur Zugänglichmachung des filmischen Erbes im DFF - Deutsches Filminstitut \& Filmmuseum
}

https://doi.org/10.1515/bfp-2020-2048

Zusammenfassung: Streaming-Angebote sind derzeit ein allgegenwärtiges Thema, doch die aktuelle Diskussion greift dabei oft zu kurz. Dieser Beitrag betrachtet grundlegende Entwicklungen und Überlegungen in der $\mathrm{Zu}$ gänglichmachung vom digitalen Bewegtbild aus der Perspektive einer filmwissenschaftlichen und filmkulturellen Institution, dem DFF - Deutsches Filminstitut \& Filmmuseum, Frankfurt am Main, und skizziert, wie sich VideoStreaming als technische Kapazität in eine Strategie zur öffentlichen Vermittlung des Filmerbes einbringt.

Schlüsselwörter: Video-Streaming; Digitalisierung; Film; Filmerbe; DFF - Deutsches Filminstitut \& Filmmuseum

\section{Moving Pictures (In A New Way)-Streaming as Part of an Encompassing Strategy to Create Access to Film Heritage}

\begin{abstract}
Streaming services are a ubiquitous topic, yet the current discourse often appears to be rather short-sighted. Taking the viewpoint of a film heritage institution - DFF Deutsches Filminstitut \& Filmmuseum - this article therefore addresses essential developments and considerations regarding the public access to digital and digitized film materials. Thereby, it also illustrates how video streaming as a technological capacity might fit into a strategy of ensuring and furthering the availability of film heritage.
\end{abstract}

Keywords: Video streaming; digitisation; film; film heritage; DFF - Deutsches Filminstitut \& Filmmuseum

\section{Einleitung}

Streaming ist mitnichten ein neues Thema, auch wenn es aktuell vor dem dramatischen Hintergrund der Covid-19Pandemie eine besonders erhöhte Aufmerksamkeit erfährt. Denn angesichts der akuten Einschränkungen öffentlicher

*Kontaktperson: David Kleingers, kleingers@dff.film
Vorführmöglichkeiten verlagert sich die Bereitstellung von Bewegtbild-Inhalten derzeit in großem Umfang in den digitalen Raum: Filmfestivals bieten Online-Editionen ihrer Programme an, internationale Produktionen starten entweder parallel zum zwangsläufig limitierten Kinoeinsatz oder gleich komplett exklusiv auf kommerziellen Plattformen, und in schneller Taktung drängen neue gewerbliche Video-Streaming-Angebote auf einen rapide wachsenden Markt, wo sie zwangsläufig immer vehementer um die limitierte Zeit - sowie die begrenzten finanziellen Ressourcen - potenzieller Nutzerinnen und Nutzer konkurrieren.

So ist auch dieses populäre Bild der „Streaming Wars“, welches momentan den Diskurs dominiert. Doch aus Sicht einer internationalen Filmerbe-Institution wie des DFF - Deutsches Filminstitut \& Filmmuseum (DFF), Frankfurt am Main, greift eine so vornehmlich auf kommerzielle Anbieter und die temporäre Marktsituation beschränkte Betrachtung zu kurz. Entsprechend seines öffentlichen Kernauftrags, nämlich die Bewahrung, Erschließung, Vermittlung und Zugänglichmachung des filmischen Erbes in all seinen Facetten zu ermöglichen und zu gewährleisten, reichen die Überlegungen des DFF zum Themenkomplex Video-Streaming nicht nur weiter zurück als die aktuelle Debatte, sie haben zugleich auch perspektivische Möglichkeiten und Herausforderungen im Blick. Im Folgenden soll es daher weniger um spezifische technische Lösungen oder individuelle Online-Angebote von Filmen gehen, sondern vielmehr um die übergeordnete Frage, wie sich Video-Streaming als Baustein in Zukunft konzeptionell in eine ganzheitliche Strategie der öffentlichen und nicht-kommerziellen Zugänglichmachung von Bewegtbild im digitalen Zeitalter fügt.

Dabei sind bereits erzielte Errungenschaften in der digitalen Dokumentation, Erschließung und Kontextualisierung des filmischen Erbes ebenso zu berücksichtigen, wie die retrospektive Digitalisierung filmischer Materialien, die standardisierte Erfassung von filmografischen sowie objektbezogenen Metadaten, der erforderliche Aufbau einer technischen Infrastruktur zur dauerhaften Vorhaltung 
und flexiblen Bereitstellung digitaler Inhalte sowie rechtliche Rahmenbedingungen. Nicht zuletzt geht es auch darum, wie sich Video-Streaming als neue bzw. komplementäre Form der Zugänglichmachung zum Selbstverständnis einer Filmerbe-Institution verhält und mit ihrer Verpflichtung gegenüber dem Kulturgut Film in Einklang gebracht wird.

\section{Dauerhafter Auftrag und digitale Transformation}

Als älteste filmwissenschaftliche Einrichtung der Bundesrepublik, die 1949 als Deutsches Institut für Filmkunde gegründet wurde und 2019 ihr 70-jähriges Bestehen begehen konnte, erfüllt das DFF gemeinsam mit den anderen beiden Einrichtungen des Kinematheksverbunds - dem Bundesarchiv und der Stiftung Deutsche Kinemathek dauerhafte Aufgaben von gesamtstaatlichem Interesse. Für die kontinuierliche Wahrnehmung dieses öffentlichen Auftrags ist es zudem erforderlich, technische Weiterentwicklungen nicht nur zu begleiten, sondern aktiv in den diversen Tätigkeitsfeldern einer Institution zu nutzen.

Diesen Anspruch reflektiert auch das Leitbild des DFF:

„Das DFF - Deutsches Filminstitut \& Filmmuseum ist eine führende internationale Filmerbe-Institution. Wir sind Vorreiter darin, das Filmerbe zu bewahren und Filmkultur mit einer weltweiten Öffentlichkeit $\mathrm{zu}$ teilen. Dafür vereinen wir auf einzigartige Weise Museum, Kino, Archive und Sammlungen, Festivals, digitale Plattformen, Forschung und Digitalisierungsprojekte sowie zahlreiche Bildungsangebote. Von der kulturell vielfältigen und dynamischen Metropole Frankfurt am Main aus arbeiten wir aktiv an der Gestaltung einer offenen Gesellschaft mit. Wir unterhalten Beziehungen zu Institutionen und Initiativen aus Film und Wissenschaft weltweit. Dabei verbinden wir die materielle und historische Bedeutung von Film mit der digitalen Zukunft.

Es ist uns Anliegen und Aufgabe, gemeinsam mit unserem Publikum Filmkultur lebendig zu halten. Wir, ein Team von mehr als 200 Mitarbeiter/innen, widmen uns dieser Mission mit Fachkenntnis und Leidenschaft. Für uns ist alles Film und Film alles."

Nicht zuletzt die digitalen Transformationsprozesse - welche andauern und sich in Zukunft fraglos immer weiter fortsetzen werden - sowie die damit einhergehende Etablierung neuer Abteilungen, Aufgaben und Angebote waren und sind mithin maßgebend für das Selbstverständnis des in den vergangenen Jahrzehnten stetig gewachsenen DFF.

1 https://www.dff.film/ueber-uns/dff/.
Entscheidend für die historische Entwicklung des DFF ist zudem die seit jeher systematisch betriebene Erfassung und Publikation von relevanten Informationen zur nationalen Filmproduktion: Seit seiner Gründung der umfassenden Dokumentation des Deutschen Films verpflichtet, hat das DFF sehr früh IT-basierte Verfahren zur Verarbeitung von filmografischen Daten eingesetzt. Bei der Datenmodellierung für seine zentrale filmografische Datenbank (ZDB) griff das DFF konzeptionell auf die Functional Requirements for Bibliographic Records (FRBR) zurück. Dieser Ansatz wurde durch die Standardisierungsinitiative „Identification of Cinematographic Works“ (2005-2009) bestätigt, deren Ergebnis in Form des vom DFF maßgeblich mitentwickelten, europäischen Standards EN 15907 vorliegt. Die erfolgreiche Entwicklung und Implementierung des Standards bildet seitdem eine wesentliche Grundlage für die Interoperabilität von filmwerksbezogenen Metadaten. $^{2}$

Mit den Fortschritten in der standardisierten filmografischen Erfassung und Dokumentation wuchs das Bestreben, neue öffentliche Zugänge im digitalen Raum zu schaffen. In dieser Hinsicht war der initiale Launch von filmportal.de ${ }^{3}$ als die öffentliche Internetplattform für den Deutschen Film im Jahr 2005 ein Meilenstein: Entwickelt und betrieben vom DFF - und dauerhaft gefördert von der Beauftragten der Bundesregierung für Kultur und Medien (BKM) und dem Land Hessen - setzt das Portal auf der zentralen filmografischen Datenbank des DFF auf und bietet so Zugriff auf fortlaufend aktualisierte und erweiterte Angaben zu allen deutschen Produktionen, von den Anfängen des Kinos bis zum aktuellen Filmstart. Aktuell hält das Portal verifizierte Daten zu über 151000 Filmwerken und mehr als 226000 Personen vor (Stand September 2020) und stellt damit die größte, öffentlich und kostenfrei zugängliche Nationalfilmografie Europas.

Im Sinne einer ganzheitlichen Dokumentation werden die Einträge seit Start des Portals zudem redaktionell durch Inhaltsangaben, Biografien, Fotos, weitere Textund Bildmaterialien sowie systematische Nachweise der öffentlichen Verfügbarkeit von Filmen ergänzt. Darüber hinaus ist filmportal.de auch Publikationsort des im Aufbau befindlichen, zentralen Bestandskatalogs deutscher Filme. So kann das Portal prinzipiell den kompletten Zyklus eines Filmwerkes abbilden- von der Produktion über die verschiedenen Formen der Veröffentlichung bis hin zur Überlieferung in den Archiven. Natürlich würde die On-

2 Weitere Informationen zu EN 15907 und zur Standardisierungsinitiative finden sich unter www.filmstandards.org.

3 https://www.filmportal.de/. 


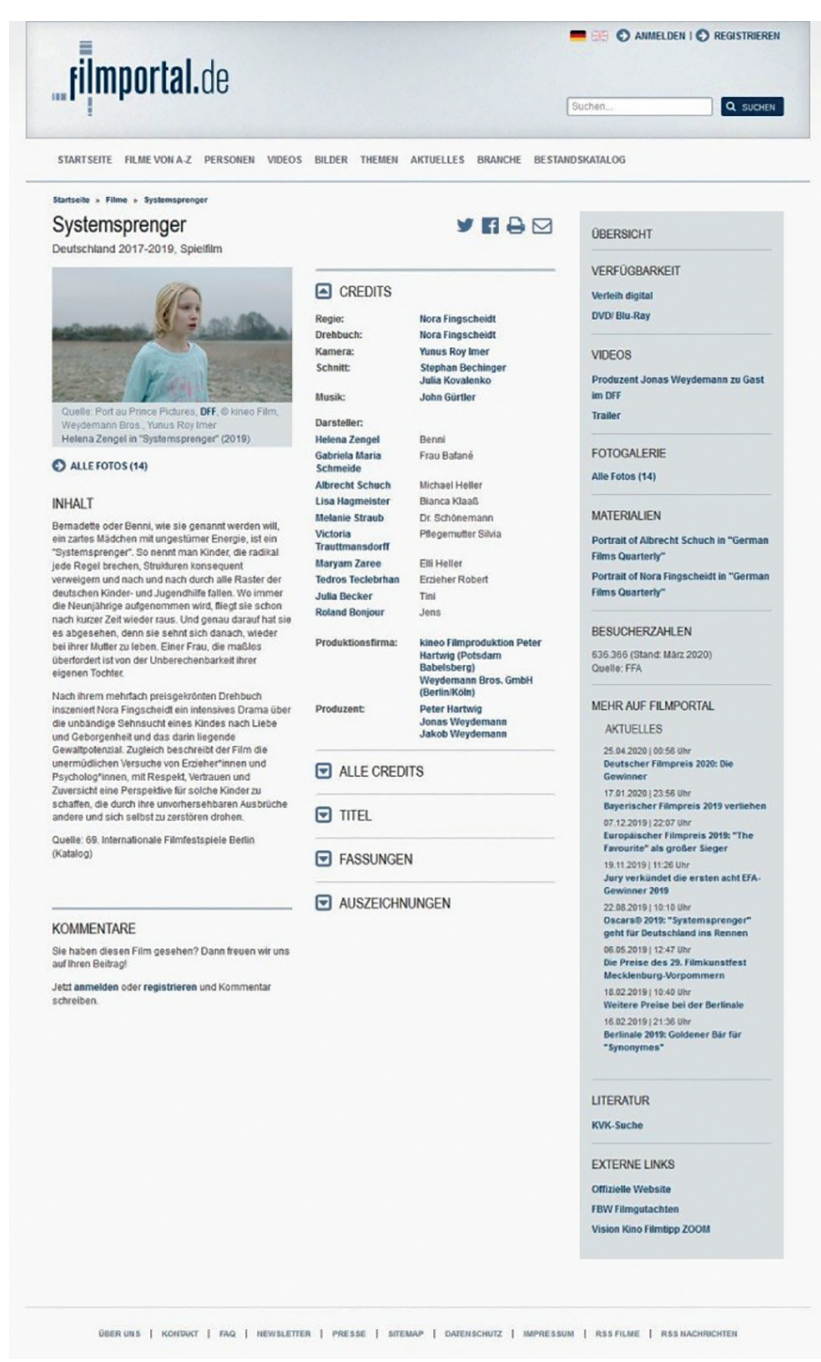

Abb. 1: Übersichtseite eines Filmeintrags auf filmportal.de

line-Zugänglichmachung digitalisierter Filme im Portal via Video-Streaming die logische Vervollständigung des öffentlichen Informationsangebots darstellen, und tatsächlich stellt filmportal.de bereits heute Video-Materialien zur Verfügung. Doch vor einer wirklich umfänglichen Zugänglichkeit des filmischen Erbes im Internet sind weitere Hürden zu überwinden, und einige davon will dieser Artikel im Weiteren näher beleuchten.

Seit der erfolgreichen Etablierung von filmportal.de als öffentlich anerkannte Referenzquelle für den Deutschen Film konnte das DFF seine Erfahrungen in zahlreiche digitale Projekte und Initiativen auf nationaler und europäischer Ebene einbringen und diese oft federführend mitgestalten: Nach der Organisationsleitung des EU-Projekts MIDAS (Moving Image Database for Access and Reuse of European Film Collections) übernahm das DFF verantwortlich die Koordination des internationalen Partnerkonsortiums zum Aufbau des European Film Gateway
(EFG), ${ }^{4}$ das den zentralen Zugriff auf digitalisierte Sammlungsbestände (Text, Foto, Film) aus mittlerweile 38 europäischen Archiven und Filmerbe-Institutionen ermöglicht und als spartenspezifischer Aggregator für die Europeana fungiert. Im Zuge des unmittelbar daran anschließenden Digitalisierungsprojekts EFG1914 wiederum konnten 740 Stunden historischen Filmmaterials aus der Zeit bzw. im Kontext des Ersten Weltkriegs erstmals online verfügbar gemacht wurden.

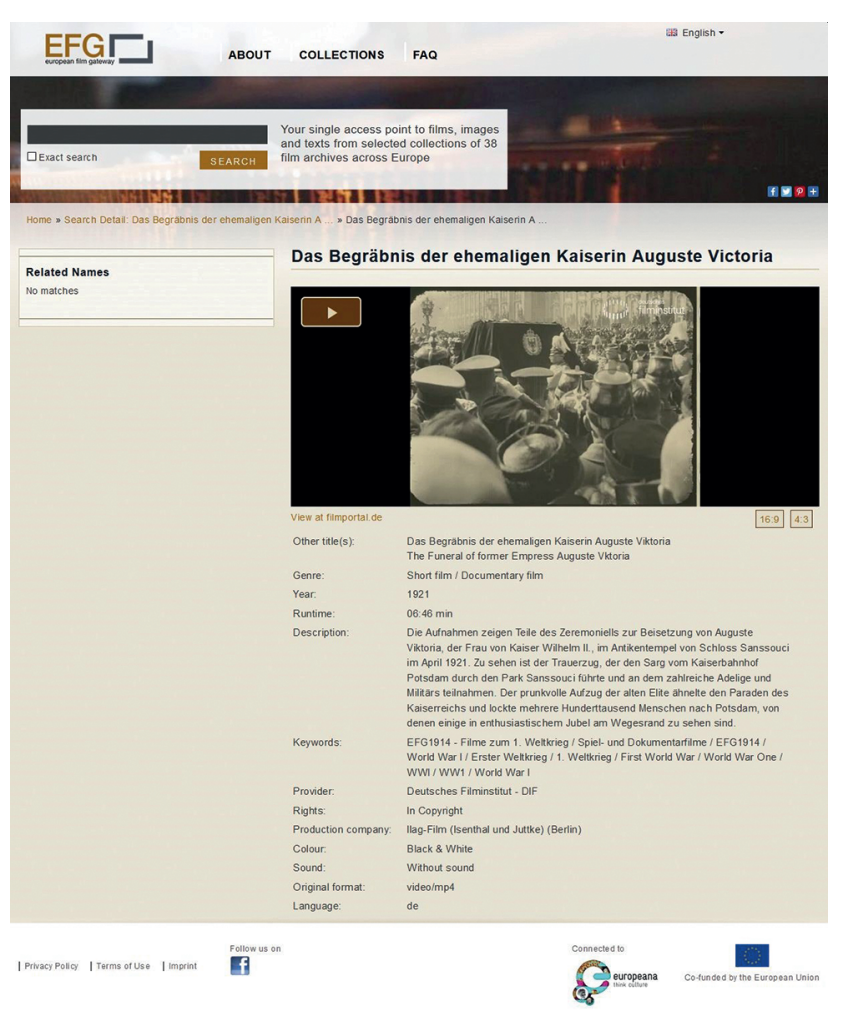

Abb. 2: Übersichtsseite zu einem im Zuge des EU-Projekts EFG1914 digitalisierten und im European Film Gateway abrufbaren Film aus den Beständen des DFF - Deutsches Filminstitut \& Filmmuseum

Auf nationaler Ebene gehörte das DFF derweil zu den Gründern der Deutschen Digitalen Bibliothek (DDB) und ist heute Spartenvertreter im Kompetenznetzwerk der DDB. Darüber hinaus übernimmt das DFF für die DDB die Aufgaben der Fachstelle der Mediatheken-Film und stellt mit filmportal.de den nationalen Aggregator für den Bereich Film.

$\mathrm{Zu}$ den jüngst abgeschlossenen bzw. aktuell laufenden digitalen Vorhaben unter Beteiligung des DFF gehören u. a. die EU-Projekte i media cities, ${ }^{5}$ Victor-E (Visual Cultu-

4 https://www.europeanfilmgateway.eu/. $5 \mathrm{https} / / /$ www.imediacities.eu/. 
re of Trauma, Obliteration and Reconstruction in PostWWII Europe $)^{6}$ und VHH-Visual History of the Holocaust. ${ }^{7}$ Weiterhin realisiert das DFF derzeit mit Förderung der Art Mentor Foundation Lucerne das Projekt RHIZOM Filmgeschichte, ${ }^{8}$ welches Formen der interaktiven Präsentation und Analyse von Filmausschnitten in einer eigenen Webapplikation erprobt. Und 2020 markierte den Start des von der Kulturstiftung des Bundes geförderten, vierjährigen Projekts Constellation 2.0 - Konstellationen filmischen Wissens, ${ }^{9}$ in dem das DFF gemeinsam mit dem Australian Center for the Moving Image (ACMI) neue Tools zur Verschränkung der Nutzererfahrungen im physischen Raum speziell in Ausstellungen - und online entwickeln will.

\section{Erschließung, Standardisierung, Vernetzung}

Eine Vielzahl dieser Projekte und Plattformen fokussiert die Publikation, Erschließung und Kontextualisierung digitaler Objekte. Fortschritte in diesem Bereich sind jedoch wesentlich abhängig von einer gleichzeitigen, kontinuierlichen Verbesserung der Datenqualität. Entsprechend hat das DFF in den vergangenen Jahren diesbezüglich ebenfalls Schritte unternommen.

So hat das DFF in Zusammenarbeit mit der Deutschen Nationalbibliothek im DFG-geförderten Pilotprojekt IN2N Institutionsübergreifende Integration von Normdaten eine Ausgabe von Personenangaben im JSON-Format der Gemeinsamen Normdatei (GND) eingerichtet. ${ }^{10}$ Ziel des Anfang 2015 abgeschlossenen Projekts war es, die Erfassung und Bearbeitung von personenbezogenen Informationen gleichzeitig in den verschiedenen Datenbanken der GND und von filmportal.de/ZDB vorzunehmen und live zu synchronisieren. Hierfür hat die Deutsche Nationalbibliothek eine neue Lese- und Schreibschnittstelle entwickelt, die sich seitdem im produktiven Betrieb durch das DFF befindet. Dies ist gleichbedeutend mit der Abkehr vom vollständigen Spiegeln der GND, die bisher Voraussetzung für die Normdatenkooperation der Verbünde darstellte, und

6 ViCTOR-E: https://www.victor-e.eu/.

7 Visual History of the Holocaust: https://www.vhh-project.eu/.

8 https://www.dff.film/erkunden/digital-international/rhizom-film geschichte/.

9 https://www.filmportal.de/nachrichten/840000-euro-foerderungfuer-digitalprojekt-von-dff-und-acmi.

10 Weitere Informationen zu dem von der Deutschen Forschungsgemeinschaft geförderten Projekt finden sich unter http://www.in2n. $\mathrm{de} /$. die solche Kooperationen weitgehend auf bibliothekarische Einrichtungen einschränkte. Mit Abschluss von IN2N sind nunmehr sämtliche Personen aus filmportal ebenfalls Teil der Gemeinsamen Normdatei. Neuerfassungen in filmportal kommen über die Schnittstelle direkt in die GND. Nebeneffekt der Kooperation ist ferner, dass die GND von ihren Personenseiten auf das entsprechende Informationsangebot auf filmportal verlinkt (und vice versa).

Im Unterschied $\mathrm{zu}$ den Stammdaten $\mathrm{zu}$ Personen, Körperschaften und Werken werden Metadaten zu digitalen Objekten, die in filmportal verfügbar sind (z. B. Bilder, Videos und weitere filmbezogene Text- und Bildmaterialien wie Produktionsunterlagen, Korrespondenz, Filmkritiken, Interviews und Filmzensurunterlagen), über eine direkt am Drupal-CMS eingerichtete Schnittstelle bereitgestellt. Dabei handelt es sich um einen OAI-PMH-Datenprovider, der die Metadaten zu digitalen Objekten bislang in einem an die Europeana Semantic Elements angelehnten Format zum Harvesting bereitstellt. Nutzer der OAIPMH-Schnittstelle sind vor allem das European Film Gateway und damit die Europeana sowie die Deutsche Digitale Bibliothek.

Im Rahmen der Neukonzeption der Exportschnittstelle von filmportal.de soll das bisher auf einem erweiterten Dublin Core beruhende Exportformat durch ein Anwendungsprofil des Europeana Data Models (EDM) ersetzt werden. Das neue RDF-basierte Exportformat ermöglicht die Referenzierung auf Linked-Open-Data-Ressourcen, wie z.B. der GND für Personen und Geografika sowie kontrollierten Vokabularen wie dem Art \& Architecture Thesaurus (AAT) oder der Library of Congress Subject Headings (LCSH). Zudem sollen Identifikatoren der entsprechenden Filmwerk-Entitäten aus Wikidata in den Export integriert werden. Diese müssen zum Teil erst durch eine vorherige Einspielung von Basis-Filmwerksdaten aus Filmportal in Wikidata generiert werden. Perspektivisch ebenfalls von Interesse wäre in diesem Kontext ein „Match and Merge“ von Filmportal-Filmwerksdaten mit GND-Werken, analog zu den Personennormdaten im IN2N Projekt, mit dem Ziel, GND-Identifikatoren zu Filmwerken ebenfalls in den filmportal-Export zu integrieren und dadurch die Vernetzung der verschiedenen Ressourcen zu unterstützen.

\section{Rechtliche Hürden der Verfügbarmachung}

Alle bislang genannten Entwicklungen und Verfahren stellen Grundlagen für eine langfristige Strategie der wirksamen Vermittlung von Film als Kulturgut im Internet 
bereit. In Anbetracht existenter und etablierter Internetplattformen, zunehmender Standardisierung und Interoperabilität von Metadaten sowie erprobter Formen der Präsentation und Kontextualisierung von digitalisierten AV-Materialien im Web lässt sich seitens der Nutzerinnen und Nutzer durchaus die Frage stellen, warum Filmerbe-Institutionen wie das DFF nicht viel mehr digitale und digitalisierte Filminhalte via Video-Streaming öffentlich und unbeschränkt verfügbar machen. Zumal wenn diese Einrichtungen oftmals umfangreiche und einzigartige Filmbestände halten. Was die Frage jedoch ausblendet, sind die weiterhin vorhandenen, wesentlichen Hürden der digitalen Zugänglichmachung von Filmen im kulturellen und nicht-kommerziellen Sektor: die z. T. prohibitiven, rechtlichen Rahmenbedingungen sowie die erheblichen Aufwände in der Digitalisierung, Vorhaltung und flexiblen Bereitstellung der Materialien.

Film ist ein immer noch junges Kultur- und Wirtschaftsgut, an das sich - gerade im Vergleich mit anderen Sparten und Kunstformen - oftmals sehr viele legitime Urheber- und Verwertungsbelange knüpfen. Filmerbe-Institutionen und Archive bewahren und erhalten die Werke der Urheberinnen und Urheber und schützen damit zugleich ihre Werte und berechtigten Interessen. Zugleich müssen sie aber auch bestrebt sein, den öffentlichen $\mathrm{Zu}$ gang zu diesen Werken so umfangreich wie rechtlich und technisch möglich zu gestalten.

Seit Jahren engagiert sich das DFF daher gemeinsam mit nationalen und europäischen Partnerinstitutionen sowie im Kinematheksverbund und der Association des Cinémathèques Européennes (ACE) für eine Verbesserung der Rechtssituation hinsichtlich der nicht-kommerziellen Verfügbarmachung von kinematographischen Werken.

So ist das DFF seit dem Start der „European Digital Library“ (heute Europeana) aktiv an Initiativen und Projekten beteiligt, die die Zugänglichkeit zum europäischen Filmerbe erleichtern sollen. Als Mitglied der High Level Expert Group on Digital Libraries (HLEG) - Subgroup Copyright ${ }^{11}$ erarbeitete Claudia Dillmann, bis September 2017 Direktorin des DFF und langjährige Präsidentin der ACE, konkrete Empfehlungen, um das kulturelle Erbe auf der Europeana-Plattform leichter verfügbar zu machen. Denn aufgrund urheberrechtlicher Regelungen in Europa drohte

11 Die High Level Experts Group on Digital Libraries wurde 2006 von EU Kommissarin Viviane Reding eingesetzt und bestand u.a. aus Vertretern des Europäischen Verlegerverbands, der Deutschen Nationalbibliothek, der British Library, der Association des Cinémathèques Européennes und Urheberrechtsexperten. Die Gruppe sprach Empfehlungen aus zu Fragen der Digitalisierung, Online-Zugänglichkeit und digitalen Bewahrung von kulturellem Material. bezogen auf die öffentliche Verfügbarkeit digitalisierten Kulturguts ein „schwarzes Loch des 20. Jahrhunderts“ zu entstehen. Dies galt insbesondere für das junge Medium Film und betraf damit auch das European Film Gateway (EFG), welches unter Federführung des DFF gemeinsam mit anderen Filmarchiven ab 2008 aufgebaut wurde.

Im Bericht der HLEG wurden verwaiste Werke als ein zentrales Problem identifiziert, denn diese Werke waren urheberrechtlich geschützt und damit für die Nutzung blockiert. Laut einer Umfrage von ACE im Jahr 2010 belief sich der Anteil verwaister Werke in den Filmarchiven Europas auf $21 \% .^{12}$ Deshalb arbeiteten das DFF wie auch die ACE intensiv auf eine europäische Regelung hin, welche die legale Nutzung verwaister Werke ermöglichen sollte. 2012 wurde eine entsprechende Regelung auch verabschiedet. ${ }^{13}$ Nach einer sorgfältigen, nachweislich erfolglosen Suche nach den Urhebern war es nun möglich, Filme für nichtkommerzielle Zwecke online zu nutzen, unter der Bedingung, dass das Werk zuvor in der EUIPO-Datenbank verzeichnet wurde. ${ }^{14}$ Parallel zum Gesetzgebungsverfahren haben die europäischen Filmarchive unter Leitung der Cinematek in Brüssel das Projekt Forward gestartet, das die Rechteklärung vereinfachen und eine Lösung für die verwaisten Filme in den europäischen Filmarchiven bereitstellen sollte. ${ }^{15}$ Während die Richtlinie die politische und gesetzliche Basis für die Nutzung lieferte, entwickelte Forward eine technische Lösung, um die sorgfältige Suche für die Filmarchive zu vereinfachen. Leider mit mäßigem Erfolg: Da die Mitgliedstaaten unterschiedliche Gesetzgebungen haben, ist eine Vereinfachung bzw. Systematisierung der sorgfältigen Suche und der Rechteklärung schwer möglich. Darüber hinaus beschränkt sich die Nutzung hier konkret das Video-Streaming - auf nicht-kommerzielle Plattformen. Das hindert viele Filmarchive daran, langwierige und aufwändige Suchen nach Urhebern durchzuführen, wenn die Aufführung/Bereitstellung im Kino, auf Festivals und auf Trägermedien wie DVD-/Blu-RayProduktion nicht erlaubt ist.

Das aktuelle Gesetzgebungsverfahren bezüglich der nicht-verfügbaren und vergriffenen Filmwerke bietet einerseits die Chance, dass es stärker angewendet wird, weil es eine zumutbare Suche nach Rechteinhabern voraus-

12 Geantwortet haben 24 Filmarchive, die Gesamtzahl der Filmwerke belief sich auf 1064000 .

13 RICHTLINIE 2012/28/EU DES EUROPÄISCHEN PARLAMENTS UND DES RATES vom 25. Oktober 2012 über bestimmte zulässige Formen der Nutzung verwaister Werke.

14 https://euipo.europa.eu/orphanworks/.

15 https://ace-film.eu/projects/forward-framework-eu-wide-audiovi sual-orphan-works-registry/. 
setzt. Andererseits sehen wir auch hier das Problem, dass die Nutzung nur online und für nicht-kommerzielle Zwecke erfolgen darf. Der Kinematheksverbund hat in einer gemeinsamen Stellungnahme zur Umsetzung der DSMRichtlinie ${ }^{16}$ auf dieses Problem hingewiesen. ${ }^{17}$

Um den Zugang zum Filmerbe also tatsächlich zu erleichtern, muss das Urheberrecht bzw. Copyright in allen Mitgliedstaaten harmonisiert werden. Bislang gibt es z.B. immer noch kein einheitliches Vorgehen, um die Schutzdauer eines Filmwerkes zu ermitteln. Während die meisten Mitgliedstaaten die „Vier-Haupturheber-Regelung“18 anwenden, gilt dies nicht für das UrhG in Deutschland - alle Beteiligten mit kreativem Input gelten potentiell als Urheber eines Filmwerks. Wenn jedoch die Frage, ob ein Film gemeinfrei ist oder nicht, nur durch komplexe Recherche ermittelt werden kann, versteht man, warum so viele Filme in den Archiven sich weiterhin im Dornröschenschlaf befinden.

\section{Sichtbarkeit durch Digitalisierung}

Bleibt noch die Hürde der erheblichen Aufwände in der Digitalisierung, Vorhaltung und Bereitstellung von Filmen $\mathrm{zu}$ betrachten. Zumindest im Bereich der retrospektiven Digitalisierung konnte nach Jahren der kulturpolitischen Diskussion in Deutschland ein entscheidender Durchbruch erzielt werden: Im Januar 2019 startete das Förderprogramm Filmerbe (FFE), gemeinsam finanziert durch die Beauftragte der Bundesregierung für Kultur und Medien (BKM), die Länder und die Filmförderungsanstalt (FFA). ${ }^{19}$ Das auf zehn Jahre angelegte Programm sieht vor, insgesamt 100 Millionen Euro - 10 Millionen Euro p.a. - für die Digitalisierung des nationalen Filmerbes bereit zu stellen. Die Vergabe der Mittel erfolgt in drei Fördersäulen, mit denen zwischen Digitalisierungsvorhaben aus kuratorischem oder konservatorischem Interesse sowie zum Zweck

16 DSM Richtlinie (EU) 2019/790 des Europäischen Parlaments und des Rates vom 17. April 2019 über das Urheberrecht und die verwandten Schutzrechte im digitalen Binnenmarkt und zur Änderung der Richtlinien 96/9/EG und 2001/29/EG.

17 Stellungnahme des Kinemathsverbundes zum Diskussionsentwurf des Bundesministeriums der Justiz und für Verbraucherschutz (BMJV) zum Zweiten Gesetz zur Anpassung des Urheberrechts an die Erfordernisse des digitalen Binnenmarktes https://www.bmjv.de/Sha redDocs/Gesetzgebungsverfahren/Stellungnahmen/2020/Download s/073120_Stellungnahme_Kinematheksverbund_RefE_UrheberrechtII.pdf?_blob=publicationFile\&v=2.

$18 \mathrm{Zu}$ den vier Haupturhebern gehören: Hauptregisseur, Drehbuchautor, Dialog-Autor, Komponist der Originalmusik.

19 https://www.ffa.de/foerderprogramm-filmerbe.html. der kommerziellen Auswertung unterschieden wird. Der deutsche Kinematheksverbund begleitet das Förderprogramm seit den ersten Überlegungen zur Ausgestaltung aktiv mit und hat die wesentlichen Erfordernisse aus Sicht der nationalen Filmerbe-Institutionen in Positionspapieren dargelegt. ${ }^{20}$

Das FFE dient dezidiert der öffentlichen (Wieder-)Zugänglichmachung des Filmerbes, d.h., es ist kein Programm zur digitalen Sicherung oder Langzeitarchivierung von Materialien. Folgerichtig schreiben die technischen Kriterien der Förderung die Erstellung kinotauglicher Digitalisierungen vor, um so nach der bereits vollzogenen Umrüstung der Lichtspieltheater auf digitale Vorführtechnik wesentliche Teile des Filmbestands wieder aufführbar zu machen. Eine Auswertung bzw. Zugänglichmachung via Video-Streaming-Angebote ist zwar nicht grundsätzlich ausgeschlossen, der Fokus des Programms liegt jedoch nachvollziehbar darauf, Filmwerke zur ursprünglich intendierten, technisch wie ästhetisch optimalen Aufführung ins Kino zu bringen.

Entsprechend der gemeinsamen Förderrichtlinie von BKM, Ländern und FFA übernimmt das DFF mit seiner Internetplattform filmportal.de die zentrale Dokumentation des Förderprogramms Filmerbe und seiner Ergebnisse. Neben der Übermittlung von relevanten Angaben zu den einzelnen Digitalisierungsvorhaben verpflichtet die Richtlinie die Förderempfänger bei Produktionen mit einer Laufzeit über 60 Minuten, die ersten fünf Minuten des digitalisierten Films dauerhaft zur öffentlichen Präsentation auf filmportal.de zur Verfügung zu stellen.

Das Verfahren der zentralen Dokumentation gewährleistet, dass sämtliche durch das Förderprogramm ermöglichten Digitalisierungen fortlaufend auf filmportal.de erfasst und recherchierbar sind. Das Video-Streaming der Filmanfänge als Teil der Dokumentation ist dabei nicht nur besonders eindrücklicher Beleg für den Erfolg des FFE, sondern trägt auch maßgeblich zur öffentlichen Wahrnehmung der digitalisierten Filme bei.

\section{Streaming-Kapazitäten für flexible Zugänge zum filmischen Erbe}

Die hier kursorisch thematisierten Entwicklungen, Erfolge und Hindernisse verdeutlichen, dass Video-Streaming aus

20 https://www.filmportal.de/nachrichten/digitalisierung-des-filme rbes-die-position-des-kinematheksverbundes. 


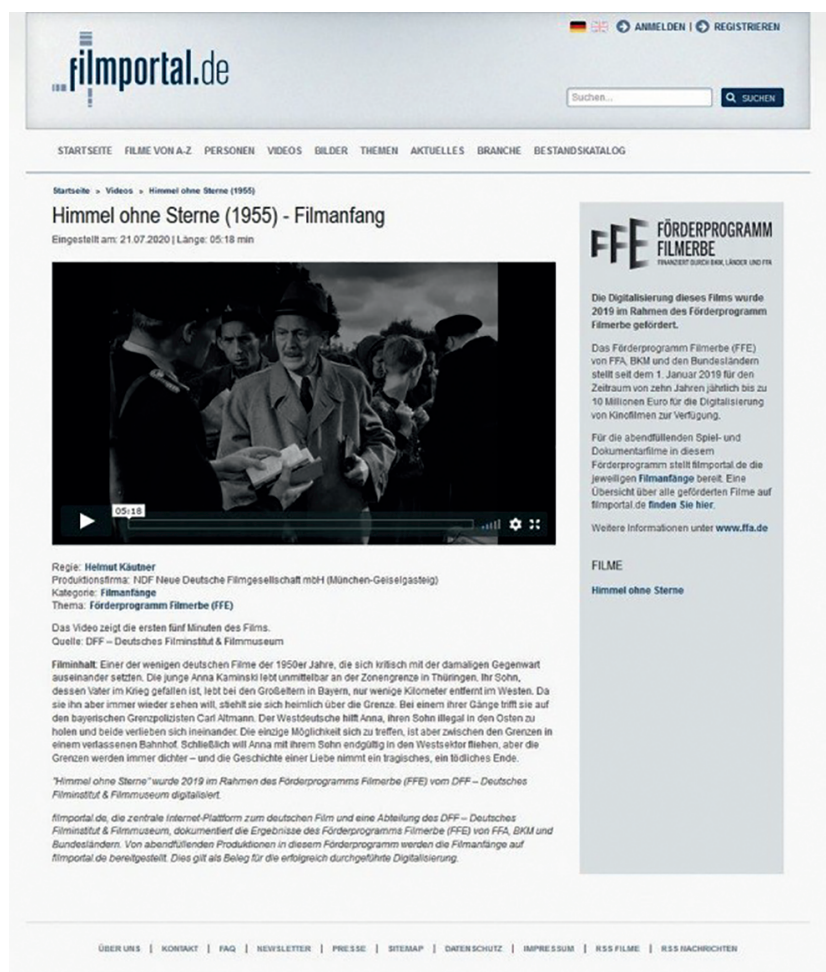

Abb. 3: Präsentation der ersten fünf Minuten eines digitalisierten Kinofilms als Teil der zentralen Dokumentation des Förderprogramms Filmerbe auf filmportal.de

Sicht einer Kultur- und Wissenseinrichtung wie dem DFF weniger im enggefassten Sinn spezifischer, singulärer Online-Angebote gedacht werden sollte, sondern vielmehr als Bestandteil einer umfassenden und langfristig tragfähigen Gesamtstrategie zur Zugänglichmachung des filmischen Erbes.

So nutzt bzw. unterhält etwa das DFF bereits Plattformen, voran filmportal.de, welche nachweislich zur sinnvoll kontextualisierten Veröffentlichung von digitalen Bewegtbild-Inhalten und Filmen geeignet sind. Auch in der Erschließung der Materialien und der Verbesserung der Datenqualität sind maßgebliche Fortschritte zu verzeichnen. Konzeptionell wie faktisch sind somit längst wesentliche Grundlagen für eine intensivere Anwendung von Video-Streaming gegeben. Die primären Herausforderungen liegen daher für Institutionen wie dem DFF in den Bereichen Digitalisierung analoger Bestände, Rechtssicherheit in der flexiblen Zugänglichmachung von Materialien sowie dem Aufbau und Unterhalt einer erforderlichen Infrastruktur zur Archivierung und Vorhaltung einer stetig wachsenden Zahl digitaler Objekte.

Insbesondere letztgenannter Punkt beschäftigt zwangsläufig alle Filmerbe-Einrichtungen mit öffentlichem Auftrag zur Sammlung und Bewahrung: Da die professionelle Filmproduktion seit Jahren fast ausschließlich digital stattfindet, sehen sich die Archive zukünftig einem exponentiellen Aufwuchs digitaler Sammlungsbestände gegenüber. Und mögen die Konzepte und Anwendungsszenarien für den öffentlichen Zugang zu diesen Beständen auch verhältnismäßig weit gediehen sein, die gleichsam notwendige Implementierung technischer Lösungen für eine skalierbare Archivierung und den variablen Playout digitaler Ressourcen ist derzeit noch ein Desiderat. Hier wird es mittel- und langfristig weitreichender Strukturmaßnahmen und substanzieller Investitionen in die digitale Infrastruktur bedürfen, damit die Einrichtungen ihren ureigenen Auftrag auch in Zukunft vollumfänglich erfüllen können.

Nicht minder relevant ist eine grundlegende Anpassung der rechtlichen Rahmenbedingungen an die Realitäten und Bedürfnisse der kulturellen Vermittlungsarbeit: Video-Streaming nur im Kontext der aktuell im Fokus stehenden Online-Plattformen zu begreifen, blendet ganz wesentliche Perspektiven der nicht-kommerziellen Nutzung von digitalem Bewegtbild in der Arbeit einer Institution wie des DFF aus. So könnte ein flexibler Playout digitalen Materials via Video-Streaming dort ebenso sinnvoll in Ausstellungen, bei den hauseigenen Filmfestivals, in der Programmierung des Kinos, bei akademischen Lehrveranstaltungen sowie in der Filmbildung und -vermittlung zur Anwendung kommen. Vorausgesetzt, die Rechtslage gestattet es der Institution, das Potenzial digitaler Ressourcen in ihren diversen Tätigkeitsfeldern und damit über die „klassische“ Online-Bereitstellung hinaus auszuschöpfen.

Darum sollte sich die Diskussion im öffentlichen Sektor perspektivisch weniger mit dem Für und Wider einzelner Streaming-Angebote befassen, sondern mit der viel grundlegenderen Frage, wie Video-Streaming als technische Kapazität für die Kultur- und Bildungsarbeit in Zukunft umfassend verfüg- und nutzbar gemacht werden kann. Speziell mit Blick auf das Filmerbe muss es bei all diesen Überlegungen darum gehen, seine öffentliche Visibilität in Zukunft zu gewährleisten. Denn nur was sichtbar ist, wird auch als einzigartiges Gut wahrgenommen.

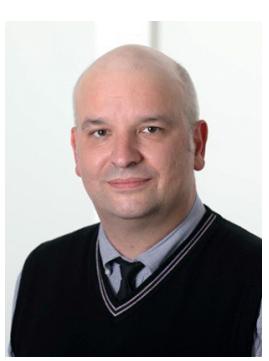

\section{David Kleingers}

DFF - Deutsches Filminstitut \& Filmmuseum

Schaumainkai 41

D-60596 Frankfurt am Main

kleingers@dff.film 